\title{
Infusion of lidocaine into the dorsal hippocampus before or after the shock training phase impaired conditioned freezing in a two-phase training task of contextual fear conditioning
}

\author{
Shih-Dar Chang, Der-Yow Chen, K. C. Liang * \\ Department of Psychology, National Taiwan University, 1 Roosevelt Road, Section 4, Taipei 106, Taiwan, ROC
}

Received 9 April 2007; revised 25 July 2007; accepted 25 July 2007

Available online 6 September 2007

\begin{abstract}
Learning in a contextual fear conditioning task involves forming a context representation and associating it with a shock. The dorsal hippocampus $(\mathrm{DH})$ is implicated in representing the context, but whether it also has a role in associating the context and shock is unclear. To address this issue, male Wistar rats were trained on the task by a two-phase training paradigm, in which rats learned the context representation on day 1 and then reactivated it to associate with the shock on day 2; conditioned freezing was tested on day 3 . Lidocaine was infused into the DH at various times in each of the two training sessions. Results showed that intra-DH infusion of lidocaine shortly before or after the context training session on day 1 impaired conditioned freezing, attesting to the DH involvement in context representation. Intra-DH infusion of lidocaine shortly before or after the shock training session on day 2 also impaired conditioned freezing. This deficit was reproduced by infusing lidocaine or APV ( $\alpha$-amino-5-phosphonovaleric acid) into the DH after activation of the context memory but before shock administration. The deficit was not due to drug-induced state-dependency, decreased shock sensitivity or reconsolidation failure of the contextual memory. These results suggest that in contextual fear conditioning integrity of the $\mathrm{DH}$ is required for memory processing of not only context representation but also context-shock association.
\end{abstract}

(c) 2007 Elsevier Inc. All rights reserved.

Keywords: Amygdala; Context-shock association; Emotional memory; Latent learning; Memory consolidation; Memory reconsolidation; NMDA receptors; Rats

\section{Introduction}

Contextual fear conditioning involves associating an otherwise neutral context with an aversive stimulus. In a typical training trial a rat is exposed to a novel environment for a period of time and then a shock is administered. Evidence has shown that this association cannot be formed if a rat has not explored the context sufficiently before receiving the shock (Fanselow, 1990). This immediate shock deficit has led to a proposition that contextual fear conditioning contains a sequence of learning processes: environmental cues encountered in a context are first inte-

\footnotetext{
* Corresponding author. Fax: +88622362 9909.

E-mail address: kcliang@ntu.edu.tw (K. C. Liang).
}

grated into a unitary representation that can be stored and retrieved subsequently; then this configural context representation is associated with the shock (Fanselow, 2000; Kiernan \& Westbrook, 1993; Rudy, Huff, \& Matus-Amat, 2004). These processes are suggested to serially engage different brain structures: the dorsal hippocampus (DH) is involved in forming and retrieving a context representation; and the amygdala, particularly the basolateral complex (BLA), is involved in associating this representation with the shock (Fanselow \& Kim, 1994; Fendt \& Fanselow, 1999; Maren \& Fanselow, 1996; Sanders, Wiltgen, \& Fanselow, 2003).

This view accounts for the findings that lesions of the DH impaired contextual fear conditioning but not cue-specific fear conditioning presumably by preventing integration of multi-modality sensory cues (Kim \& Fanselow, 
1992; Phillips \& LeDoux, 1992), but lesions of the BLA impaired both kinds of fear conditioning presumably by preventing association of the conditioned stimulus or context with the shock (Kim, Rison, \& Fanselow, 1993; Phillips \& LeDoux, 1992). However, this view is inconsistent with some of the existing data. A previous study has shown that after extensive training, rats bearing BLA lesions could not acquire cue-specific conditioned freezing but could still acquire contextual conditioned freezing (Maren, 1999a). Spared context-shock association after BLA lesions was also reported in tasks of Pavlovian fear conditioning (Cahill, Vazdarjanova, \& Setlow, 2000) or inhibitory avoidance learning (Berlau \& McGaugh, 2003). These findings suggest that under certain circumstances brain structures other than the BLA may also be involved in associating the context and shock.

As the DH has long been implicated in coding stimulus relationship (Eichenbaum, Otto, \& Cohen, 1992), its role in associating the context and shock was suggested by the previous evidence that hippocampal lesions impaired certain forms of conditioned fear expressed in a context including defecation or body temperature change that was impervious to amygdala lesions (Antoniadis \& McDonald, 2000, 2001; Sutherland \& McDonald, 1990). This conjecture was corroborated by recent findings that the shock experience in various aversive learning tasks altered the firing pattern or induced experience-dependent neural plasticity in the DH neurons (Moita, Rosis, Zhou, LeDoux, \& Blair, 2004; Whitlock, Heynen, Shuler, \& Bear, 2006). These findings raise a possibility that the DH could be involved in processing context-shock association in addition to context representation.

A role of the DH in context-shock association during contextual fear conditioning may be obscured by the typical training paradigm in which acquisition of context representation and context-shock association occurs almost simultaneously within a single training session, hence independent manipulation of the various processes inherent in the task will be very difficult if not totally impossible. To address this issue, context learning and context-shock association must be temporally detached, as accomplished by some previous studies with a two-phase training paradigm of contextual fear conditioning (Fanselow, 1990; Rudy \& O'Reilly, 2001). This paradigm pre-exposed a rat on the first day to the conditioning context without administering shock to form a latent contextual memory that was reactivated on the next day, by a brief re-exposure or a reminder cue, to associate with a shock. By altering the $\mathrm{DH}$ function at the context training session, a role of the DH in context representation was confirmed (Anagnostaras, Gale, \& Fanselow, 2001; Matus-Amat, Higgins, Barrientos, \& Rudy, 2004; Rudy, Barrientos, \& O'Reilly, 2002). However, few studies to date have done a critical test with this paradigm on whether the $\mathrm{DH}$ is involved in processing the context-shock association. The present study thus employed the two-phase training paradigm to examine the effects of suppressing the DH with lidocaine at the context training or context-shock association session on conditioned freezing.

\section{Materials and methods}

\subsection{Subjects}

Male Wistar rats were obtained from Animal Center of National Taiwan University, Taipei, Taiwan and housed individually with free access to food and water. The vivarium was maintained on a 12-h light-dark cycle (light on from 9:00 am to 9:00 pm) with $22-25^{\circ} \mathrm{C}$ ambient temperature and $60-70 \%$ relative humidity. Experiments were carried out in the light phase. The animal care and experimental procedure followed Guidelines for Animal Research of Agriculture Council, ROC; and approved by Institutional Animal Care and Use Committee of National Taiwan University.

\subsection{Surgery}

All rats were subjected to stereotaxic surgery when they weighed approximately $400 \mathrm{~g}$. They were treated with atropine sulfate $(0.4 \mathrm{mg} /$ $\mathrm{kg}$ ) to prevent respiratory congestion and then anesthetized with sodium pentobarbital ( $45 \mathrm{mg} / \mathrm{kg}$, i.p.). The anesthetized rat was mounted on a stereotaxic instrument (Model 900, David Korpf Instrument, Tijuna, USA) and a midline incision was made on the scalp to expose the skull. Two 23-gauge stainless steel guide cannulae $(10 \mathrm{~mm})$ were implanted bilaterally into the $\mathrm{DH}(-3.8 \mathrm{~mm}$ posterior to bregma, $\pm 2.5 \mathrm{~mm}$ lateral to midline, $-2.2 \mathrm{~mm}$ ventral to the surface of skull; Paxinos \& Watson, 1986). Three jewelry screws serving as anchors were implanted over the frontal and posterior cortices. The whole assembly was affixed onto the skull with dental cement. A stylet of $10 \mathrm{~mm}$ was inserted into each cannula after surgery to maintain patency. Rats recuperated in their home cages for at least 7 days. They were handled 1 min per day for 5 days before an experiment.

\subsection{Contextual fear conditioning: A two-phase training paradigm}

The two-phase training paradigm of contextual fear conditioning contained a Context session and a Context/Shock session accomplished in two successive days, while the test was held on the third day (Kiernan \& Westbrook, 1993). Briefly, a conditioning box $(30 \times 24 \times 24 \mathrm{~cm}$; MED Associates Inc., East Fairfield, USA) designated as Context A was used for fear conditioning throughout this study and housed in a sound/light attenuation chamber $(60 \times 40 \times 58 \mathrm{~cm})$ situated in a dimly lit room. A 10 -W incandescent light bulb for illumination was mounted on the left chamber wall and a fan for ventilation and generation of masking noise was mounted on the back wall. The box was cleaned with $75 \%$ alcohol after each use.

In the Context session, a rat was carried from its home cage to Context A and allowed $2 \mathrm{~min}$ of free exploration and then returned to its home cage. In the Context/Shock session on the second day, the rat returned to Context $\mathrm{A}$ and $20 \mathrm{~s}$ later a footshock $(0.75 \mathrm{~mA}, 2 \mathrm{~s})$ was administered. The shock was delivered from a programmable shocker (Model VT 05448; MED Associates Inc., East Fairfield, USA) connected to the bar grid floor of Context A. The rat returned to its home cage immediately after receiving the shock. On the third day conditioned freezing was tested in Context A. The rat was videotaped for a 6-min test period, during which behavior was sampled every $4 \mathrm{~s}$. Two independent raters blind to the treatment judged at each sampled time whether the rat showed freezing behavior that was defined as absence of any movement except for respiration; the percentage of freezing was used as an index of conditioned fear (Fanselow, 1982). Across experiments an inter-rater reliability over .95 was achieved. A pilot study showed that this procedure yielded robust conditioned freezing behavior in our laboratory.

\subsection{A reminder cue procedure for the two-phase training paradigm}

Some experiments in this study adopted a reminder cue procedure (Rudy \& O'Reilly, 2001) as follows: in the Context session on the first day, a rat was transported from its home cage to Context A by Chamber 
$\mathrm{X}$ carried on a cart, which took about $20 \mathrm{~s}$. Chamber X was a white translucent plastic cage $(45 \times 25 \times 15 \mathrm{~cm})$ filled with sawdust on the floor and covered with plywood at the top. Once entering the context, the rat was allowed free exploration for 2 min at this first stay. It was then carried back to its home cage by Chamber X and stayed there for $40 \mathrm{~s}$ before the next transportation. This procedure was repeated four additional times and each time the rat stayed in Context A for only $40 \mathrm{~s}$. In the Context/ Shock session on the second day, the rat was transported by Chamber $\mathrm{X}$ from its home cage to Context $\mathrm{A}$ and received a footshock immediately (within a second or so) after entering the context. On the third day, the rat was transported by its home cage to Context $\mathrm{A}$ for assessing freezing. This procedure allowed the transportation chamber to become a reminder cue that activated the full context representation at the Context/Shock session. Literature has shown that the context memory activated as such could readily associate with the shock and yield robust conditioned freezing, even though the shock was administered at a time when the rat might not yet fully recognize the context (Rudy et al., 2002; Rudy et al., 2004).

\subsection{Shock-induced startle: A test for shock sensitivity}

To evaluate possible effects of the administered drugs on shock sensitivity, rats were placed into the startle apparatus and subjected to a shock/ acoustic startle test (Chen, Ho, \& Liang, 2000). The startle apparatus (San Diego Instrument, San Diego, USA) consisted of a Plexiglas cylindrical container with a vibration sensor at its base, and was enclosed in a ventilated sound/light attenuation chest $(30 \times 38 \times 55 \mathrm{~cm})$. In a test session, a rat was placed into the container under a continuous $55 \mathrm{~dB}$ background noise. After an acclimation period of $5 \mathrm{~min}$, it received 45 startle trials with 30 -s inter-trial intervals. The stimuli were either 40 -ms white noise bursts at 95,105 and $115 \mathrm{~dB}$ or 100 -ms electric shocks in 9 different intensities ranging from 0 to $1.6 \mathrm{~mA}$ in a $0.2-\mathrm{mA}$ step. Each session had three blocks of trial, each block contained 6 acoustic trials ( 2 trials for each sound level) followed by 9 shock trials ( 1 trial for each shock level). The startle response was measured by the vibration sensor for a period of $200 \mathrm{~ms}$ after the stimulus onset and digitized by a computer for further analyses. This paradigm could assess independently effects of a treatment on sensitivity and motor reactivity to a stimulus. For a treatment specifically affecting shock sensitivity, an effect should appear only in shock startle but not in acoustic startle. However, if the treatment affected the startle ability in general, both forms of startle would change.

\subsection{Drug infusion}

Lidocaine hydrochloride (Sigma, St. Louis, USA) or D,L-2-amino-5phosphonovaleric acid (APV; Tocris, Bristol, UK), an $N$-methyl-D-aspartate (NMDA) receptor antagonist, was dissolved into phosphate-buffered saline ( $\mathrm{pH}$ 7.4). The concentration of lidocaine was $4 \%(\mathrm{w} / \mathrm{v})$, which has been shown to effectively inactivate the DH (Hammond, Tull, \& Stackman, 2004; Packard \& McGaugh, 1996). The dose of APV was 2.5 or $5.0 \mu \mathrm{g}$ for each side of the DH. Infusion was accomplished by inserting a 30-gauge infusion needle into the guide cannula with the needle extending $1 \mathrm{~mm}$ beyond cannula the tip. Infusion of either drug or vehicle was administered simultaneously to both sides of the $\mathrm{DH}$ at a rate of $0.5 \mu \mathrm{l} /$ min with a syringe pump (CMA/100, Carnegie Medicin, Stockholm, Sweden). A total volume of $0.5 \mu \mathrm{l}$ were infused into each side. After infusion, the needle stayed in the cannula for $1 \mathrm{~min}$ before being withdrawn. One day before the training session, all rats assigned to an infusion group were acclimated with the above procedure except for nothing being infused.

\subsection{Histology}

At the conclusion of each experiment, the implanted rats were anesthetized with an overdose of anesthetics and perfused through the heart with saline followed by $10 \%$ formalin. The brain was removed and stored in formalin solution with $20 \%$ sucrose for at least 7 days. Frozen sections $(40 \mu \mathrm{m})$ throughout the cannula tract were mounted on slides
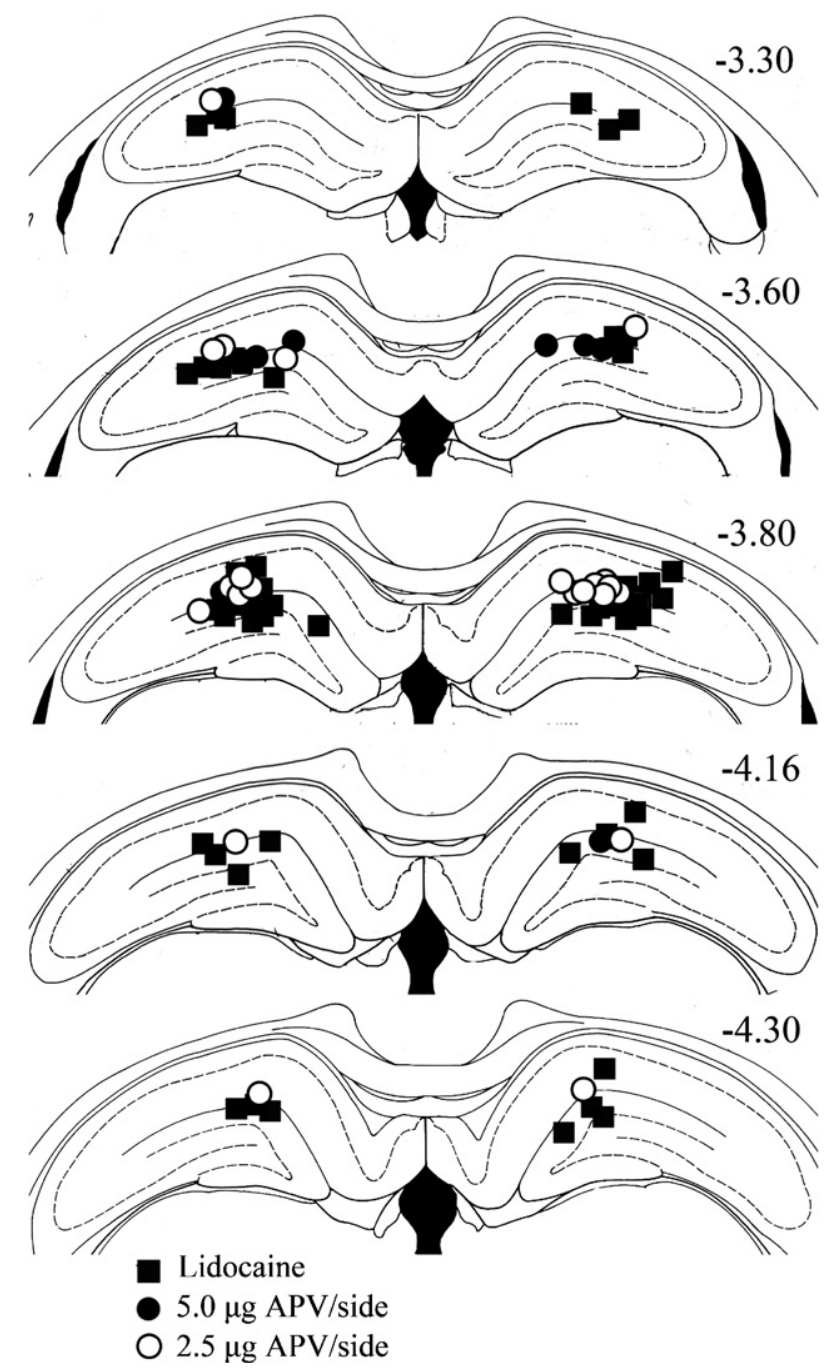

Fig. 1. Distribution of infusion needle tips in the dorsal hippocampus for a sample of experimental animals. The coronal brain plates are adapted from the atlas of Paxinos and Watson (1986).

and stained with thionin. Only animals with both cannulae correctly placed in the DH were accepted. Fig. 1 shows the distribution of cannula tips in a sample (51 out of 264) of all experimental animals included in the final data analysis.

\section{Results}

\subsection{Inactivating the DH at the Context or Context/Shock session impaired conditioned freezing}

This experiment examined effects of inactivating the $\mathrm{DH}$ at various times during the two learning phases on conditioned freezing. Rats were assigned randomly to different groups to receive bilateral intra-DH infusion of vehicle or lidocaine at the following times: before the Context session, after the Context session, or before the Context/Shock session (as shown in Fig. 2a). The vehicle group $(n=12)$ received vehicle at all three occasions. The pre-Context group $(n=12)$ received lidocaine $5 \mathrm{~min}$ prior to the Context session but vehicle at the other two occasions. 
a
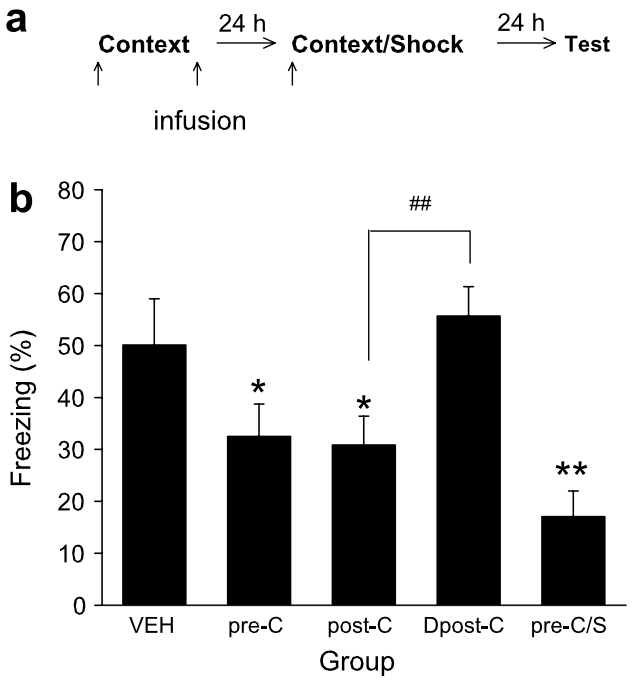

Fig. 2. Effects of suppressing the dorsal hippocampus with lidocaine at various times on conditioned freezing during the 6-min test session. (a) A schematic illustration of the experimental procedure. (b) Percentage of freezing (mean $\pm \mathrm{SEM}$ ) during the test session. Rats received intra-dorsal hippocampal infusion of lidocaine shortly before the Context session (Group pre-C), immediately after the Context session (Group post-C), $6 \mathrm{~h}$ after the Context session (Group Dpost-C), or shortly before the Context/ Shock session (Group pre-C/S). The vehicle group (Group VEH) received vehicle on all three occasions. ${ }^{*} p<.05,{ }^{* *} p<.01$, significantly lower than Group VEH. ${ }^{\#} p<.01$, significantly lower than Group Dpost-C.

The 5-min time point was chosen because lidocaine did not reach its full effect until 3 min after administration (Martin, 1991). The immediate post-Context group $(n=12)$ received lidocaine immediately after the Context session and vehicle at the other two occasions. The delayed post-Context group $(n=12)$ received lidocaine 6-h after the Context session and vehicle at the other two occasions. The immediate and $6 \mathrm{~h}$ time points were chosen because aversive memory has been shown to consolidate within this time window (Izquierdo \& Medina, 1997). The pre-Context/Shock group $(n=12)$ received lidocaine $5 \mathrm{~min}$ prior to the Context/ Shock session and vehicle at the other two occasions. During the Context/Shock session, all groups of rats were placed again into Context $\mathrm{A}$ and $20 \mathrm{~s}$ later a footshock was administered.

Fig. 2b shows the mean scores of conditioned freezing for the five groups: intra-DH infusion of lidocaine shortly before or after the Context session impaired conditioning, but the delayed infusion had no effect. Intra-DH infusion of lidocaine given shortly before the Context/Shock session also impaired conditioned freezing. A one-way ANOVA revealed a significant difference among the five groups $\left(F_{(4,55)}=6.64 ; p<.01\right)$. Post hoc analyses with the Fisher LSD test indicated that the vehicle group exhibited more conditioned freezing than the pre-Context, immediate post-Context, and pre-Context/Shock groups $(p<.05, .05$ and .01 ; respectively), but the three lidocaine-treated groups did not differ among themselves $(p>.05)$. The mean freezing score of the delayed post-Context group was significantly higher than that of the immediate post-Context group $(p<.01)$ but did not differ from that of the vehicle group.

To evaluate whether the deficits caused by lidocaine given before either training session were due to statedependency, two additional groups of rats received bilateral intra-DH infusion of lidocaine $(n=11)$ or vehicle $(n=11)$ before the Context, Context/Shock and test sessions. The averaged score of conditioned freezing was $59.69 \pm 6.45 \%$ (mean $\pm \mathrm{SEM}$ ) for the vehicle group and $36.46 \pm 4.52 \%$ for the lidocaine group. A one-way ANOVA revealed a significant difference between the two groups $\left(F_{(1,20)}=8.51 ; p<.01\right)$. These results did not support a state-dependency interpretation for the observed deficit.

\subsection{Inactivating the DH immediately after the Contextl Shock session impaired conditioned freezing}

Given the above finding that suppressing the DH before the Context/Shock session impaired conditioned freezing, this experiment further tested whether the same treatment given after the Context/Shock session interfered with context-shock association memory and caused a time-dependent deficit in conditioned freezing. Four groups of rats were trained with the procedure described in Section 2.3 and received bilateral intra-DH infusion of vehicle or lidocaine either immediately or $6 \mathrm{~h}$ after the Context/Shock session. Fig. 3 shows the mean freezing scores in the test for the immediate and delayed infusion groups: lidocaine impaired conditioned freezing in rats receiving the immediate infusion but not in rats receiving the delayed infusion. Two one-way ANOVAs revealed a significant difference between the two immediate infusion groups
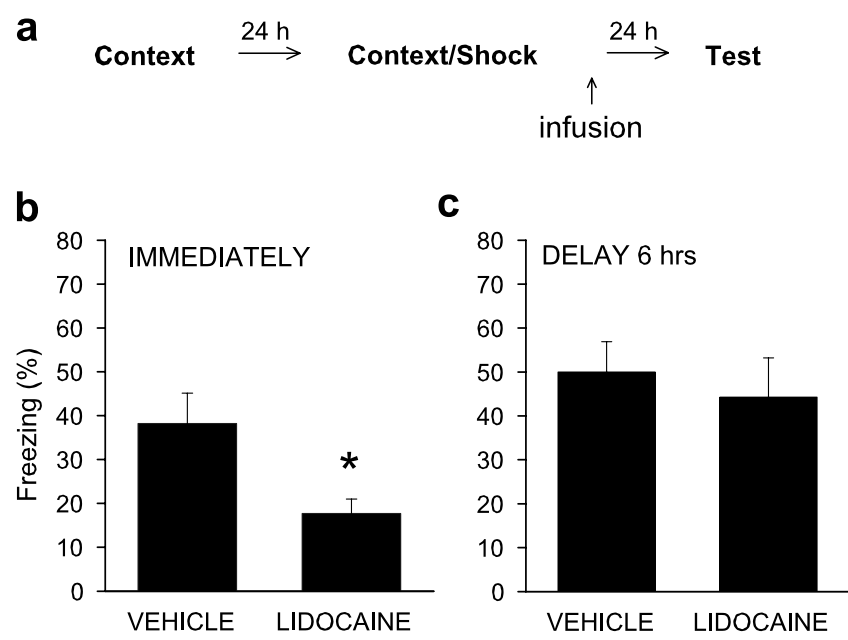

Fig. 3. Effects of suppressing the dorsal hippocampus with lidocaine immediately or $6 \mathrm{~h}$ after the Context/Shock session on conditioned freezing. (a) A schematic illustration of the experimental procedure. (b) Percentage of freezing (mean $\pm \mathrm{SEM}$ ) in the groups receiving infusion immediately after the Context/Shock session. (c) Percentage of freezing (mean $\pm \mathrm{SEM}$ ) in the groups receiving infusion $6 \mathrm{~h}$ after the Context/ Shock session. ${ }^{*} p<.05$, significantly lower than the group receiving vehicle. 
$\left(F_{(1,23)}=6.76 ; p<.05\right)$, but not between the two delayed infusion groups $\left(F_{(1,22)}<1\right)$. Thus, inactivating the $\mathrm{DH}$ after the Context/Shock session induced a time-dependent retention deficit in conditioned freezing.

The deficit induced by lidocaine infused after the shock could be due to disruption of re-storage processing, such as reconsolidation, of the activated contextual memory. This possibility was tested by examining the effect of DH suppression after a trial involving only retrieval and re-storage of the contextual memory but no shock association. If the deficit observed above was due to disrupted re-storage, such manipulation would hinder the association formation in a subsequent Context/Shock session and yield the same deficit in a later test. However, if the above deficit was indeed due to interference with context-shock association, this manipulation would cause no effect. Two additional groups of rats were subjected to the two-phase training paradigm modified as follows: on day 1 , rats were exposed to Context A; on day 2, the contextual memory was activated by putting the rat back into Context $\mathrm{A}$ for $20 \mathrm{~s}$ without administration of shock. Immediately following this reexposure, rats received bilateral intra-DH infusion of lidocaine $(n=8)$ or vehicle $(n=9)$. On day 3 , the rat was put into Context $\mathrm{A}$ and $20 \mathrm{~s}$ later it received a shock. On day 4 , all rats were tested for conditioned freezing in Context A. The mean freezing scores at the test were $55.42 \pm 4.57 \%$ for the vehicle group and $49.14 \pm 7.27 \%$ for the lidocaine group. A one-way ANOVA showed no difference between the two groups $\left(F_{(1,15)}<1\right)$. These data showed that suppressing the DH immediately but not $6 \mathrm{~h}$ after the Context/Shock session impaired conditioned freezing. On the other hand, suppressing the DH immediately after a pure retrieval trial involving no context-shock association caused no effect. Thus the treatment impaired consolidation of the context-shock memory rather than reconsolidation of the context memory.

\subsection{Inactivating the DH during shock administration in a reminder cue procedure impaired conditioned freezing in long-term but not short-term memory tests}

In view of the findings in Section 3.2, the deficit induced by intra-DH infusion before the Context/Shock session observed in Section 3.1 could be due to impairment in retrieval of the context memory and/or association of this memory to the shock. Potential contribution of the latter process to the observed effect could be tested more specifically by the reminder cue procedure described in Section 2.4 that enabled us to examine whether intra-DH infusion of lidocaine after retrieving a well-formed context memory but before associating it to the shock would affect shortterm memory (STM) and long-term memory (LTM) of conditioned freezing. Three groups of rats were subjected to the reminder cue training procedure and tested for conditioned freezing immediately and 1-day after training with a protocol depicted schematically in Fig. 4a. Rats in the control group $(n=13)$ were transported from their home
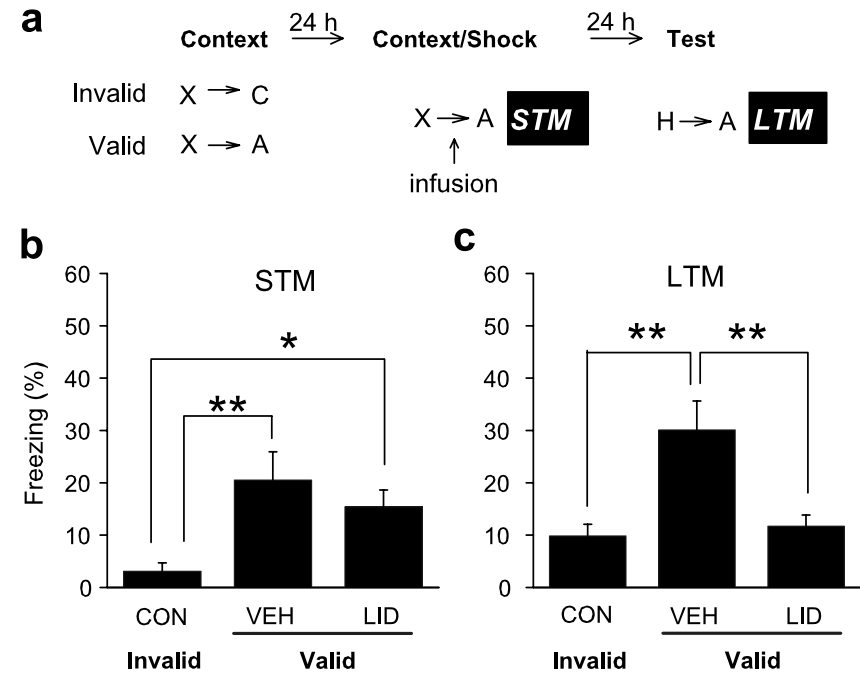

Fig. 4. Differential effects of suppressing the dorsal hippocampus with lidocaine after presentation of the reminding cue but before administration of the shock on conditioned freezing in an immediate test (STM) and a 1-day test (LTM). (a) A schematic illustration of the experimental procedure. The control group $(\mathrm{CON})$ received the invalid conditioning procedure and intra-DH infusion of vehicle, the vehicle (VEH) and lidocaine (LID) groups received the valid conditioning procedure and intra-DH infusion of vehicle and lidocaine, respectively. (b) Percentage of freezing (mean \pm SEM) during the STM test in the Context/Shock session. (c) Percentage of freezing (mean \pm SEM) during the LTM test. ${ }^{*} p<.05$, ${ }^{* *} p<.01$

cages by Chamber $\mathrm{X}$ to explore in Context $\mathrm{C}$, which was a compartment $(35 \times 35 \times 35 \mathrm{~cm})$ in a shuttle box with features and location site different from Context A described previously. In contrast, the vehicle $(n=13)$ and lidocaine $(n=13)$ groups were transported from their home cages by Chamber X to Context A. At the Context/Shock session rats in all groups were transported by Chamber $\mathrm{X}$ to Context $\mathrm{A}$, and after being removed from Chamber $\mathrm{X}$ but before entering Context $\mathrm{A}$ to have shock they received bilateral intra-DH infusion of vehicle or lidocaine. Immediately after being placed into Context $\mathrm{A}$, the rat received a foot shock. Five seconds later, STM was evaluated in Context A by assessing freezing behavior for $30 \mathrm{~s}$ in every $2 \mathrm{~s}$. One day later, LTM for all rats was evaluated in Context A by a 6-min normal test as previously described. For the control group, Chamber $\mathrm{X}$ was never paired with Context A beforehand, thus in the Context/Shock session this cue was unable to activate the representation of Context A to associate with the shock administered immediately after entry. Thus, the control group received invalid conditioning and its freezing behavior could serve as a no-association baseline, against which STM and LTM of the vehicle and lidocaine groups receiving valid conditioning could be compared.

Fig. $4 \mathrm{~b}$ and $\mathrm{c}$ shows the mean freezing scores of the three groups in the STM and LTM tests. Two one-way ANOVAs revealed significant differences among the three groups in the STM and LTM tests $\left(F_{(2,36)}=5.68\right.$ and 9.41, respectively; $p<.01)$. Post hoc analyses with the 
Fisher LSD test indicated that in the STM test, the vehicle and lidocaine groups receiving valid conditioning expressed significantly more freezing than the control group receiving invalid conditioning $(p<.05)$, but the former two groups did not differ between themselves ( $p>.10)$, suggesting that the vehicle and lidocaine groups equally acquired the association. In the LTM test, while the vehicle group receiving valid conditioning still showed significantly more freezing than the control group receiving invalid conditioning $(p<.01)$, the lidocaine group receiving valid conditioning failed to do so and showed freezing significantly lower than the vehicle group $(p<.01)$. These results showed that suppressing the $\mathrm{DH}$ after retrieving context memory but before context-shock association did not affect conditioned freezing in the STM test but impaired it in the LTM test.

To address the issue that the observed deficit was indeed due to interference with consolidation of context-shock association rather than reconsolidation of the activated context memory, two additional groups of rats were subjected to a modified reminder cue procedure as follows: all rats received the standard day- 1 procedure depicted in Section 2.4. On day 2, rats were carried by Chamber X to the conditioning room and then received bilateral intra-DH infusion of lidocaine $(n=8)$ or vehicle $(n=8)$ immediately after removal from Chamber X. After the infusion, they returned directly to their home cages. On day 3, all rats were taken from its home cage to Context A by Chamber $\mathrm{X}$ to receive an immediate shock. On day 4 , all rats were transported by their home cages to Context A for testing. The mean freezing scores were $36.53 \pm 5.41 \%$ for the vehicle group and $45.69 \pm 9.05 \%$ for the lidocaine group. A one-way ANOVA revealed no statistical difference between the two $\left(F_{(1,14)}<1\right)$. These results were not consistent with the idea that the deficit induced by intraDH infusion of lidocaine within a Context/Shock session in the reminder cue procedure could be due to interference with reconsolidation of the context memory per se.

\subsection{Intra-DH infusion of $A P V$ in the reminder cue procedure impaired conditioned freezing}

To pursue further whether association of context and shock indeed required the $\mathrm{DH}$, we examined the effect on conditioned freezing of blocking hippocampal NMDA receptors that were implicated in induction but not expression of experience-driven neural plasticity in the DH (Collingridge, Kehl, \& McLennan, 1983). Three groups of rats were trained with the reminder cue procedure as described schematically in Fig. 5a. At the Context/Shock session, after being removed from Chamber $\mathrm{X}$, rats received bilateral intra-DH infusion of vehicle $(n=19), 2.5 \mu \mathrm{g}$ APV $(n=10)$ or $5.0 \mu \mathrm{g}$ APV $(n=11)$. After the infusion, they were placed into Context $\mathrm{A}$ and received a shock immediately. Fig. $5 \mathrm{~b}$ shows the results that intra-DH infusion of APV caused a dose-dependent deficit in conditioned freezing. A one-way ANOVA revealed a significant difference among the groups $\left(F_{(2,37)}=4.32 ; p<.05\right)$. Post hoc analy-
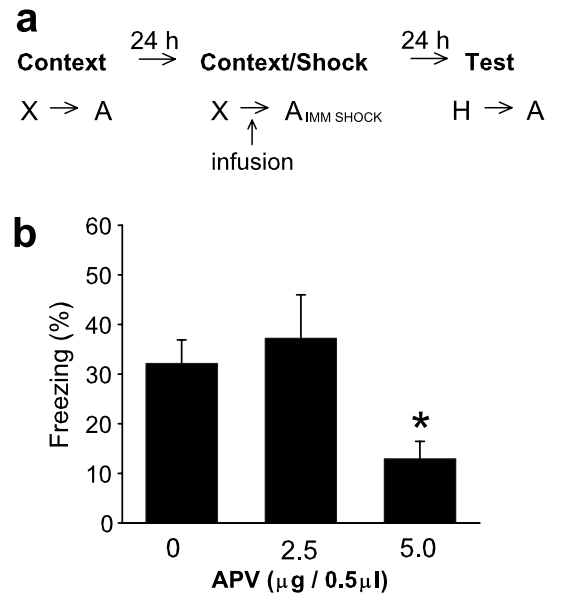

Fig. 5. Effects of APV infused into the dorsal hippocampus after presentation of the reminding cue but before administration of the shock on conditioned freezing. (a) A schematic illustration of the experimental procedure. IMM SHOCK: shock administered immediately after entering the context; $\mathrm{H}$ : home cage. (b) Percentage of freezing (mean $\pm \mathrm{SEM}$ ) during the 6-min test session for rats receiving infusion of vehicle $(0 \mu \mathrm{g})$, $2.5 \mu \mathrm{g}$ or $5.0 \mu \mathrm{g}$ APV $(0.5 \mu \mathrm{l}$ per side) into the dorsal hippocampus ${ }^{*} p<.05$, significantly lower than the vehicle group.

ses with the Dunnett test revealed that the $5.0 \mu \mathrm{g}$ group displayed less freezing than the vehicle group $(p<.05)$.

To ascertain whether this learning-impairing dose of APV had any effect on retrieval, two additional groups of rats were trained with the two-phase training procedure (Section 2.3) and received bilateral intra-DH infusion of vehicle or $5.0 \mu \mathrm{g} \mathrm{APV} \mathrm{shortly} \mathrm{before} \mathrm{being} \mathrm{tested} \mathrm{on} \mathrm{the}$ third day. The mean freezing scores over the 6-min testing period were $35.45 \pm 7.99 \%$ for the vehicle group and $39.44 \pm 10.29 \%$ for the APV group. One-way ANOVAs revealed no statistical difference between the two groups $\left(F_{(1,19)}<1\right)$. Because in the experiment described above the shock was administered shortly after APV infusion, integrity of memory expression at an early phase after APV infusion would be most critical for forming a normal association. The minute-by-minute freezing score was scrutinized over the test period for possible difference between the vehicle and APV groups. No significant difference between the two groups was detected in expression of conditioned freezing by planned $t$-tests for the first or any following minute of the entire test (all ps>.10).

\subsection{Intra-hippocampal infusion of lidocaine or APV did not affect shock sensitivity}

To evaluate whether intra-DH infusion of $4 \%$ lidocaine or $5.0 \mu \mathrm{g}$ APV shortly before shock administration affected the shock sensitivity, a sample of rats was subjected to a shock startle test at least 7 days after the conditioning task. Rats from the groups in Section 3.1 were divided randomly to receive intra-DH infusion of vehicle $(n=9)$ or lidocaine $(n=9)$. Rats from the groups in Section 3.4 were also randomly divided to receive intra-DH infusion of vehicle 


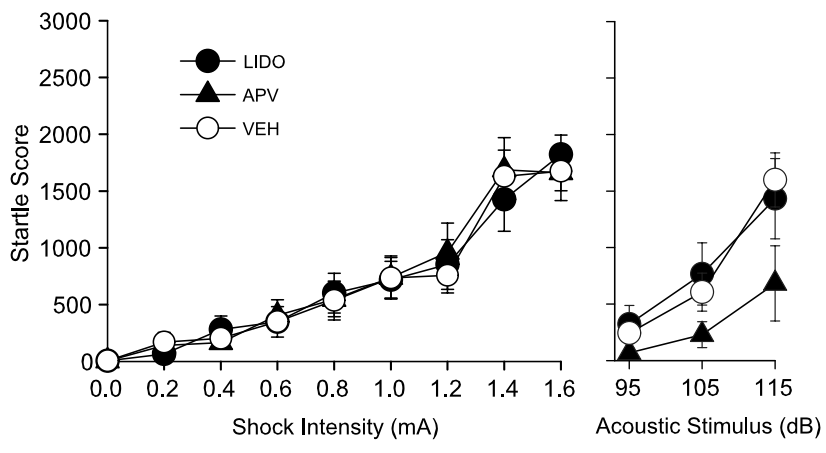

Fig. 6. Lack of effect of lidocaine or APV on the startle amplitude (mean \pm SEM) induced by shock (left panel) and acoustic stimuli (right panel). Three groups of rats received intra-dorsal hippocampal infusion of lidocaine (LIDO), APV (5.0 $\mu \mathrm{g}$ per side) or vehicle (VEH). No statistically significant difference among the three groups was detected in either form of startle.

$(n=9)$ or $5.0 \mu \mathrm{g}$ APV $(n=10)$. Because lidocaine caused a total blockade of the target area within 3-15 min and the effect subsided gradually in an hour (Malpeli, 1999; Martin, 1991), the first two blocks of the startle responses elicited by shock and acoustic stimuli were analyzed. The two vehicle groups showed no difference in either shock or acoustic startle, thus their data were pooled into a single control group. Fig. 6 shows the mean amplitude of startle response elicited by different intensities of shock and acoustic stimuli: intra-DH infusion of lidocaine failed to alter shock or acoustic startle. While the APV-treated rats appeared to have lower acoustic startle scores than the control or lidocaine group, two-way ANOVAs (Drug $\times$ Stimulus-Intensity) revealed no statistical significance in either the shock startle or acoustic startle for the Drug main effect $\left(F_{(2,34)}<1\right.$ for the shock data; $F_{(2,34)}=2.24 ; p>.05$ for the acoustic data) and Drug $\times$ Stimulus-Intensity interaction $\left(F_{(16,272)}<1\right.$, for the shock data; $F_{(2,34)}=2.08 ; p>.05$, for the acoustic data). The Stimulus-Intensity main effect was significant $\left(F_{(8,272)}=54.7, p<.01\right.$ for the shock data; $F_{(2,68)}=39.19, p<.01$ for the acoustic data), suggesting that for all three-treated groups, both acoustic and shock startle responses increased along with elevation of the stimulus intensity.

\section{Discussion}

To assess the DH roles in context learning and contextshock association, this study used a training paradigm that separates the two learning processes in contextual fear conditioning. Consistent with previous results (Kiernan \& Westbrook, 1993; Rudy \& O'Reilly, 2001), our data showed that the context information could be acquired without explicit reinforcement and stored in dormancy until being activated for further association. Thus, context learning in the present study is essentially a kind of latent learning noted in other behavioral tasks by previous studies (Liang, 1999; Noda et al., 2001; Tolman, 1948). Results from this latent learning task suggest that the $\mathrm{DH}$ is involved in formation of memory for both processes.

The present study probed the DH function with lidocaine that has an effect vanishing in an hour (Malpeli, 1999; Martin, 1991). Thus, its influence could be confined to a specific phase of memory processing rather than affecting both memory formation and expression as electrolytic lesions (Phillips \& LeDoux, 1992; Rudy et al., 2002). For infusion experiments, whether the observed effect reflects a drug action at the infusion site is of great concern. In view of that $1 \mu \mathrm{l}$ of $4 \%$ lidocaine inactivated a tear-drop shape area within $1.5 \mathrm{~mm}$ of the infusion needle tip (Martin, 1991), $0.5 \mu 1$ used in this study should inactivate an even smaller region encroaching the $\mathrm{DH}$ without invading much of the adjacent tissue. Treatments given before a training session may exert effects on performance rather than learning. However, in our study posttraining treatments without influencing sensory or motivational states during acquisition induced similar effects as pretraining ones, thus the observed deficits should be due to defects in learning and memory per se (McGaugh, 2000). The lack of a lidocaine or APV effect on shock startle argues more specifically against a contribution of altered shock sensitivity to the observed deficits. Our data, in line with previous findings (Matus-Amat et al., 2004), also ruled out a statedependency account for the lidocaine effect.

The results that lidocaine infused into the $\mathrm{DH}$ before the Context session impaired conditioned freezing confirm the previous suggestion for involvement of the $\mathrm{DH}$ in acquiring contextual memory (Anagnostaras et al., 2001; Fanselow, 2000). Our results also showed that this treatment given after the session caused a time-dependent deficit, consistent with a notion that the $\mathrm{DH}$ is involved in consolidation of the context memory (Biedenkapp \& Rudy, 2004; Malin \& McGaugh, 2006) but conflicting with that intraDH infusion of muscimol after context training did not impair conditioned freezing (Matus-Amat et al., 2004). The cause for this discrepancy is not readily clear but may be due to the fact that muscimol engages only GABA-mediated inhibition, yet lidocaine suppresses all neural activity. Beta-noradrenergic activation has been shown to overcome GABA inhibition in modulating avoidance memory (Introini-Collison, Castellano, \& McGaugh, 1994). Thus, lidocaine may eliminate all memory enabling factors including those counteracting GABA inhibition, such as norepinephrine that was implicated in contextual fear conditioning (Frankland et al., 2004; Ji, Wang, \& Li, 2003). Alternatively, lidocaine may exert its effect by directly inhibiting intracellular cAPM production and hence protein kinase A activation (Onozuka, Watanabe, Imai, Nagasaki, \& Yamamoto, 1993; Roux et al., 1989), which played a crucial role in formation of contextual fear memory (Schafe, Nadel, Sullivan, Harris, \& LeDoux, 1999).

The most critical findings of this study are that perturbing the $\mathrm{DH}$ at the Context/Shock session impaired conditioned freezing. The impairment induced by intra-DH 
infusion of lidocaine before the session could be due to deficits in retrieving the context memory as suggested by a previous study applying muscimol into the DH (Matus-Amat et al., 2004). Yet as the lidocaine effect lasted into the postshock period, the treatment might also affect processing of context-shock association. The deficit induced by intraDH infusion lidocaine after the Context/Shock session attested to this conjecture. The time-dependent nature of this impairment, namely the immediate post-shock infusion inducing a marked deficit but the delayed infusion inducing no deficit, is consistent with the interpretation that the treatment affected the memory consolidation process of context-shock association. While our data cannot rule out a possibility that lidocaine given after the shock affected memory processing of shock per se rather than that of context-shock association, such an interpretation was not supported by the findings that manipulating the $\mathrm{DH}$ did not interfere with fear conditioning to a specific cue (Kim \& Fanselow, 1992; Phillips \& LeDoux, 1992).

Additional findings in a reminder cue procedure of the present study provide evidence consistent with, though not necessarily proving, the above suggestion. Suppressing the DH after retrieval of the contextual memory but before its association with the shock significantly impaired freezing behavior tested 1 day after training but had no effect on that tested within 1 min after shock administration. The negligible freezing in an invalid-conditioning control group (Fig. 4b) supports the notion that in contextual fear conditioning, freezing present shortly after the shock relied on the context-shock association and thus was a conditioned response (Fanselow, 1982, 1986). The normal STM but impaired LTM of conditioned freezing in the lidocaine-treated group is consistent with the notion that suppressing the DH could prevent the context-shock association from consolidating.

Previous studies also reported that disturbing the DH functions in various ways did not impair STM of conditioned freezing, and attributed this to association of the shock to a set of individual elements sampled in exploring the context (Kim et al., 1993; Sanders \& Fanselow, 2003; Young, Bohenek, \& Fanselow, 1994). This interpretation, however, may not account for our results, because in a reminder cue procedure a rat had little time to sample elements of the context as the shock appeared immediately upon its entering the context. An intact STM of contextshock association in our lidocaine group suggests that integrated context memory, once activated under normal $\mathrm{DH}$ functions, may not be repressed by subsequent suppression of this structure. Such a view, while awaits more evidence to sustain, is compatible with a theory that the memory may be stored in the cortex but require the $\mathrm{DH}$ only for its formation and retrieving at an early stage (Squire \& Alvarez, 1995; Teyler \& Discenna, 1986).

Based on the findings that that intra-cerebroventricular infusion of APV impaired acquisition but not expression of conditioned freezing (Kim, Decola, Landeira-Fernandez, \& Fanselow, 1991) and that APV did not affect formation and expression of STM in such a task (Sanders \& Fanselow, 2003), this study infused APV into the DH just before the shock in a reminder cue procedure and found a dosedependent deficit. These findings not only indicate that the observed deficit was due to compromise of neural operation within the DH per se, but also suggest that the NMDA receptors were involved in formation of long-term context-shock memory. These findings are complementary to the ones that these receptors were also involved in formation of long-term context memory (Stote \& Fanselow, 2004). While our study found no effect of APV on memory expression activated by the context, a former study showed that intra-DH infusion of APV blocked expression of conditioned fear to a tone in trace conditioning (Quinn, Loya, Ma, \& Fanselow, 2005). Given the similarity shared by the reminder cue procedure and trace conditioning in associating a shock with the image of a stimulus, the issue of whether APV blocked expression of a context memory activated by a reminder cue should be pursued in the future by a better designed experiment.

Our data showed that lidocaine given immediately after a pure contextual memory retrieval trial in the two training procedures failed to impair subsequent conditioned freezing. These results suggest that the effect induced by lidocaine given at the Context/Shock session could not be due to deficits in re-storage of the contextual memory. Evidence has suggested that a memory trace once being activated may undergo a reconsolidation process in re-storage (Nader, Schafe, \& LeDoux, 2000), which may engage different neural processes in the DH than consolidation (Taubenfeld, Milekic, Monti, \& Alberini, 2001). Our data, in line with that intra-DH infusion of anisomycin after a retrieval trial failed to affect reconsolidation of context memory (Biedenkapp \& Rudy, 2004), might not resolve those issues regarding the boundaries under which reconsolidation of contextual fear memory occurs and what neural processes in the $\mathrm{DH}$ are involved (McGaugh, 2004b; Tronson \& Taylor, 2007); however, they rendered an alternative interpretation unlikely that the observed deficit in consolidation of context-shock association could be due to reconsolidation failure of the context memory.

The present results, taken together, suggest that the $\mathrm{DH}$ has roles in memory processing for context-shock association in addition to context representation, although they do not rule out that suppressing the DH before a Context/ Shock session may hamper the association by blocking retrieval of context memory. This suggestion is consistent with the evidence that hippocampal place cells altered their place fields after administration of shock in contextual fear conditioning (Moita et al., 2004) as well as in an inhibitory avoidance task footshock training induced NMDA-dependent long-term potentiation in CA1 neurons (Whitlock et al., 2006) and the calcium-independent activity of the DH calcium calmodulin-dependent protein kinase II implicated in neural plasticity (Tan \& Liang, 1996). The findings that certain immediate early genes in the DH were activated specifically to context-shock association but others 
were induced by merely exposure to the context (Hall, Thomas, \& Everitt, 2000; von Hertzen \& Giese, 2005) also supports involvement of the $\mathrm{DH}$ in both context representation and context-shock association.

While it remains to be tested in paradigms other than latent learning of contextual fear conditioning, a role of the $\mathrm{DH}$ in context-shock association is not unexpected in lieu of the evidence that the DH may represent spatial and temporal relationship for encountered stimuli (Bangasser, Waxler, Santollo, \& Shors, 2006; Wallenstein, Eichenbaum, \& Hasselmo, 1998). However, it needs to be reconciled with some conflicting findings. The reports that pre- or posttraining electrolytic lesions of the $\mathrm{DH}$ did not abolish contextual fear conditioning if rats had been preexposed to the context a month ago (Anagnostaras et al., 2001; Young et al., 1994) imply no need for the DH in context-shock association. Yet in view of an idea that processing of contextual memory by the DH is only time-limited (Anagnostaras, Maren, \& Fanselow, 1999; Kim \& Fanselow, 1992, but see Sutherland et al., 2001 for a different view), association of a remote contextual memory with shock could engage structures other than the DH. A former study reported that anisomycin impaired conditioned freezing if given after the pre-exposure but had no effect if given after the shock (Barrientos, O'Reilly, \& Rudy, 2002). In view of the evidence that context representation and context-shock association activated different immediate early genes and thus syntheses of different proteins (von Hertzen \& Giese, 2005), the two learning processes may have different sensitivity to protein synthesis inhibition that could not be revealed by one dose of the drug given at one time point. In an inhibitory avoidance task, intra-DH infusion of oxotremorine improved memory if given after the pre-exposure but had no effect if given after the shock (Malin \& McGaugh, 2006). These findings, taken together with ours, may suggest that integrity of the $\mathrm{DH}$ is necessary for formation of the context memory and context-shock association memory, but cholinergic activation of the DH is only sufficient for enhancing the context memory as the two memories may engage different neurochemical systems in the DH.

Most studies in the literature ascribe the function of context-shock association to the BLA (Gale et al., 2004; Kim et al., 1993; Phillips \& LeDoux, 1992). While our findings are able to accommodate results incongruent with this prevailing model, they by no means disprove it. Whether suppressing the DH completely eliminated or partially attenuated conditioned freezing is inconclusive in our data, because except in Section 3.3 a no-association (or noshock) baseline of freezing was not available for comparison. It is possible that the BLA and DH are both contained in the circuitry and interacting to subserve context-shock association. The BLA may affect contextual fear conditioning via its influences on the DH (Huff \& Rudy, 2004; LaLumiere, Buen, \& McGaugh, 2003; McGaugh, 2004a), as suggested by the evidence that manipulating the BLA function modulated electrophysiological or biochemical mark- ers of neural plasticity in the DH (Almaguer-Melian, Martinez-Marti, Frey, \& Bergado, 2003; Frey, BergadoRosado, Seidenbecher, Pape, \& Frey, 2001; Huff et al., 2006; Kim, Lee, Han, \& Packard, 2001). Alternatively, the DH may affect the context-shock association by modulating processes in the BLA (Maren \& Fanselow, 1995; Maren \& Hobin, 2007; Mello, Tan, \& Finch, 1992) or the two structures may provide critical inputs converging onto a third region implicated in contextual fear conditioning, such as the ventral hippocampus (Donley, Schulkin, \& Rosen, 2005; Maren, 1999b). These views that may not be mutually exclusive remain to be tested in the future.

\section{Acknowledgments}

This study was supported by Grants NSC 92-2413-H-002015, 93-2752-H-002-006-PAE and NSC 95-2413-H002-028MY3 from the National Science Council of Taiwan, ROC. We thank C. Y. Wang and P. F. Chien for their assistance in part of the study.

\section{References}

Almaguer-Melian, W., Martinez-Marti, L., Frey, J. U., \& Bergado, J. A. (2003). The amygdala is part of the behavioural reinforcement system modulating long-term potentiation in rat hippocampus. Neuroscience, 119, 319-322.

Anagnostaras, S. G., Gale, G. D., \& Fanselow, M. S. (2001). Hippocampus and contextual fear conditioning: Recent controversies and advances. Hippocampus, 11, 8-17.

Anagnostaras, S. G., Maren, S., \& Fanselow, M. S. (1999). Temporally graded retrograde amnesia of contextual fear after hippocampal damage in rats: Within-subjects examination. Journal of Neuroscience, 19, 1106-1114.

Antoniadis, E. A., \& McDonald, R. J. (2000). Amygdala, hippocampus and discriminative fear conditioning to context. Behavioural Brain Research, 108, 1-19.

Antoniadis, E. A., \& McDonald, R. J. (2001). Amygdala, hippocampus, and unconditioned fear. Experimental Brain Research, 138, 200-209.

Bangasser, D. A., Waxler, D. E., Santollo, J., \& Shors, T. J. (2006). Trace conditioning and the hippocampus: The importance of contiguity. Journal of Neuroscience, 26, 8702-8706.

Barrientos, R. M., O'Reilly, R. C., \& Rudy, J. W. (2002). Memory for context is impaired by injecting anisomycin into dorsal hippocampus following context exploration. Behavioural Brain Research, 134, 299-306.

Berlau, D. J., \& McGaugh, J. L. (2003). Basolateral amygdala lesions do not prevent memory of context-footshock training. Learning \& Memory, 10, 495-502.

Biedenkapp, J. C., \& Rudy, J. W. (2004). Context memories and reactivation: Constraints on the reconsolidation hypothesis. Behavioral Neuroscience, 118, 956-964.

Cahill, L., Vazdarjanova, A., \& Setlow, B. (2000). The basolateral amygdala complex is involved with, but is not necessary for, rapid acquisition of Pavlovian 'fear conditioning'. European Journal of Neuroscience, 12, 3044-3050.

Chen, D. Y., Ho, S. H., \& Liang, K. C. (2000). Startle responses to electric shocks: Measurement of shock sensitivity and effects of morphine, buspirone and brain lesions. Chinese Journal of Physiology, 43, 35-47.

Collingridge, G. L., Kehl, S. J., \& McLennan, H. (1983). Excitatory amino-acids in synaptic transmission in the schaffer collateral commissural pathway of the rat hippocampus. Journal of Physiology, 334, 33-46. 
Donley, M. P., Schulkin, J., \& Rosen, J. B. (2005). Glucocorticoid receptor antagonism in the basolateral amygdala and ventral hippocampus interferes with long-term memory of contextual fear. Behavioural Brain Research, 164, 197-205.

Eichenbaum, H., Otto, T., \& Cohen, N. J. (1992). The hippocampus: What does it do. Behavioral and Neural Biology, 57, 2-36.

Fanselow, M. S. (1982). The postshock activity burst. Animal Learning \& Behavior, 10, 448-454.

Fanselow, M. S. (1986). Associative vs. topographical accounts of the immediate shock freezing deficit in rats: Implications for the response selection rules governing species-specific defensive reactions. Learning and Motivation, 17, 16-39.

Fanselow, M. S. (1990). Factors governing one-trial contextual conditioning. Animal Learning \& Behavior, 18, 264-270.

Fanselow, M. S. (2000). Contextual fear, gestalt memories, and the hippocampus. Behavioural Brain Research, 110, 73-81.

Fanselow, M. S., \& Kim, J. J. (1994). Acquisition of contextual pavlovian fear conditioning is blocked by application of an NMDA receptor antagonist D,L-2-amino-5-phosphonovaleric acid to the basolateral amygdala. Behavioral Neuroscience, 108, 210-212.

Fendt, M., \& Fanselow, M. S. (1999). The neuroanatomical and neurochemical basis of conditioned fear. Neuroscience and Biobehavioral Reviews, 23, 743-760.

Frankland, P. W., Josselyn, A., Anagnostaras, S. G., Kogan, J. H., Takahashi, E., \& Silva, A. J. (2004). Consolidation of CS and US representations in associative fear conditioning. Hippocampus, 14, $557-569$.

Frey, S., Bergado-Rosado, J., Seidenbecher, T., Pape, H. C., \& Frey, J. U. (2001). Reinforcement of early long-term potentiation (early-LTP) in dentate gyrus by stimulation of the basolateral amygdala: Heterosynaptic induction mechanisms of late-LTP. Journal of Neuroscience, 21, 3697-3703.

Gale, G. D., Anagnostaras, S. G., Godsil, B. P., Mitchell, S., Nozawa, T., Sage, J. R., et al. (2004). Role of the basolateral amygdala in the storage of fear memories across the adult lifetime of rats. Journal of Neuroscience, 24, 3810-3815.

Hall, J., Thomas, K. L., \& Everitt, B. J. (2000). Rapid and selective induction of BDNF expression in the hippocampus during contextual learning. Nature Neuroscience, 3, 533-535.

Hammond, R. S., Tull, L. E., \& Stackman, R. W. (2004). On the delaydependent involvement of the hippocampus in object recognition memory. Neurobiology of Learning and Memory, 82, 26-34.

Huff, N. C., Frank, M., Wright-Hardesty, K., Sprunger, D., Matus-Amat, P., Higgins, E., et al. (2006). Amygdala regulation of immediate-early gene expression in the hippocampus induced by contextual fear conditioning. Journal of Neuroscience, 26, 1616-1623.

Huff, N. C., \& Rudy, J. W. (2004). The amygdala modulates hippocampus-dependent context memory formation and stores cue-shock associations. Behavioral Neuroscience, 118, 53-62.

Introini-Collison, I. B., Castellano, C., \& McGaugh, J. L. (1994). Interaction of GABAergic and beta-noradrenergic drugs in the regulation of memory storage. Behavioral and Neural Biology, 61, 150-155.

Izquierdo, I., \& Medina, J. H. (1997). Memory formation: The sequence of biochemical events in the hippocampus and its connection to activity in other brain structures. Neurobiology of Learning and Memory, 68, 285-316.

Ji, J. Z., Wang, X. M., \& Li, B. M. (2003). Deficit in long-term contextual fear memory induced by blockade of beta-adrenoceptors in hippocampal CA1 region. European Journal of Neuroscience, 17, 1947-1952.

Kiernan, M. J., \& Westbrook, R. F. (1993). Effects of exposure to a to-beshocked environment upon the rat's freezing response: Evidence for facilitation, latent inhibition, and perceptual learning. Quarterly Journal of Experimental Psychology Section B-Comparative and Physiological Psychology, 46, 271-288.

Kim, J. J., Decola, J. P., Landeira-Fernandez, J., \& Fanselow, M. S. (1991). $N$-Methyl-D-aspartate receptor antagonist APV blocks acquisition but not expression of fear conditioning. Behavioral Neuroscience, $105,126-133$.
Kim, J. J., \& Fanselow, M. S. (1992). Modality-specific retrograde amnesia of fear. Science, 256, 675-677.

Kim, J. J., Lee, H. J. J., Han, J. S., \& Packard, M. G. (2001). Amygdala is critical for stress-induced modulation of hippocampal long-term potentiation and learning. Journal of Neuroscience, 21, 5222-5228.

Kim, J. J., Rison, R. A., \& Fanselow, M. S. (1993). Effects of amygdala, hippocampus, and periaqueductal gray lesions on short- and long-term contextual fear. Behavioral Neuroscience, 107, 1093-1098.

LaLumiere, R. T., Buen, T. V., \& McGaugh, J. L. (2003). Post-training intra-basolateral amygdala infusions of norepinephrine enhance consolidation of memory for contextual fear conditioning. Journal of Neuroscience, 23, 6754-6758.

Liang, K. C. (1999). Pre- or post-training injection of buspirone impaired retention in the inhibitory avoidance task: Involvement of amygdala 5HT1A receptors. European Journal of Neuroscience, 11, 1491-1500.

Malin, E. L., \& McGaugh, J. L. (2006). Differential involvement of the hippocampus, anterior cingulate cortex, and basolateral amygdala in memory for context and footshock. Proceedings of the National Academy of Sciences USA, 103, 1959-1963.

Malpeli, J. G. (1999). Reversible inactivation of subcortical sites by drug injection. Journal of Neuroscience Methods, 86, 119-128.

Maren, S. (1999a). Neurotoxic basolateral amygdala lesions impair learning and memory but not the performance of conditional fear in rats. Journal of Neuroscience, 19, 8696-8703.

Maren, S. (1999b). Neurotoxic or electrolytic lesions of the ventral subiculum produce deficits in the acquisition and expression of pavlovian fear conditioning in rats. Behavioral Neuroscience, 113, 283-290.

Maren, S., \& Fanselow, M. S. (1995). Synaptic plasticity in the basolateral amygdala induced by hippocampal formation stimulation in vivo. Journal of Neuroscience, 15, 7548-7564.

Maren, S., \& Fanselow, M. S. (1996). The amygdala and fear conditioning: Has the nut been cracked? Neuron, 16, 237-240.

Maren, S., \& Hobin, J. A. (2007). Hippocampal regulation of contextdependent neuronal activity in the lateral amygdala. Learning \& Memory, 14, 318-324.

Martin, J. H. (1991). Autoradiographic estimation of the extent of reversible inactivation produced by microinjection of lidocaine and muscimol in the rat. Neuroscience Letters, 127, 160-164.

Matus-Amat, P., Higgins, E. A., Barrientos, R. M., \& Rudy, J. W. (2004). The role of the dorsal hippocampus in the acquisition and retrieval of context memory representations. Journal of Neuroscience, 24, 2431-2439.

McGaugh, J. L. (2000). Memory - A century of consolidation. Science, 287, 248-251.

McGaugh, J. L. (2004a). The amygdala modulates the consolidation of memories of emotionally arousing experiences. Annual Review of Neuroscience, 27, 1-28.

McGaugh, J. L. (2004b). Memory reconsolidation hypothesis revived but restrained: Theoretical comment on Biedenkapp and Rudy (2004). Behavioral Neuroscience, 118, 1140-1142.

Mello, L., Tan, A. M., \& Finch, D. M. (1992). Convergence of projections from the rat hippocampal formation, medial geniculate and basal forebrain onto single amygdaloid neurons: An in vivo extra- and intracellular electrophysiological study. Brain Research, 587, $24-40$.

Moita, M. A. P., Rosis, S., Zhou, Y., LeDoux, J. E., \& Blair, H. T. (2004). Putting fear in its place: Remapping of hippocampal place cells during fear conditioning. Journal of Neuroscience, 24, 7015-7023.

Nader, K., Schafe, G. E., \& LeDoux, J. E. (2000). Fear memories require protein synthesis in the amygdala for reconsolidation after retrieval. Nature, 406, 722-726.

Noda, A., Noda, Y., Kamei, H., Ichihara, K., Mamiya, T., Nagai, T., et al. (2001). Phencyclidine impairs latent learning in mice: Interaction between glutamatergic systems and sigma(1) receptors. Neuropsychopharmacology, 24, 451-460.

Onozuka, M., Watanabe, K., Imai, S., Nagasaki, S., \& Yamamoto, T. (1993). Lidocaine suppresses the sodium current in euhadra neurons 
which is mediated by cAMP-dependent protein-phosphorylation. Brain Research, 628, 335-339.

Packard, M. G., \& McGaugh, J. L. (1996). Inactivation of hippocampus or caudate nucleus with lidocaine differentially affects expression of place and response learning. Neurobiology of Learning and Memory, $65,65-72$.

Paxinos, G., \& Watson, C. (1986). The rat brain in stereotaxic coordinates. Orlando: Academic Press.

Phillips, R. G., \& LeDoux, J. E. (1992). Differential contribution of amygdala and hippocampus to cued and contextual fear conditioning. Behavioral Neuroscience, 106, 274-285.

Quinn, J. J., Loya, F., Ma, Q. D., \& Fanselow, M. S. (2005). Dorsal hippocampus NMDA receptors differentially mediate trace and contextual fear conditioning. Hippocampus, 15, 665-674.

Roux, S., Escoubet, B., Friedlander, G., Legrimellec, C., Bertrand, I., \& Amiel, C. (1989). Effects of lidocaine on sarcolemmal fluidity and cellular cAMP in rat cardiomyocytes. American Journal of Physiology, 256, $\mathrm{H} 422-\mathrm{H} 427$.

Rudy, J. W., Barrientos, R. M., \& O'Reilly, R. C. (2002). Hippocampal formation supports conditioning to memory of a context. Behavioral Neuroscience, 116, 530-538.

Rudy, J. W., Huff, N. C., \& Matus-Amat, P. (2004). Understanding contextual fear conditioning: Insights from a two-process model. Neuroscience and Biobehavioral Reviews, 28, 675-685.

Rudy, J. W., \& O'Reilly, R. C. (2001). Conjunctive representations, the hippocampus, and contextual fear conditioning. Cognitive, Affective, \& Behavioral Neuroscience, 1, 66-82.

Sanders, M. J., \& Fanselow, M. S. (2003). Pre-training prevents context fear conditioning deficits produced by hippocampal NMDA receptor blockade. Neurobiology of Learning and Memory, 80, 123-129.

Sanders, M. J., Wiltgen, B. J., \& Fanselow, M. S. (2003). The place of the hippocampus in fear conditioning. European Journal of Pharmacology, $463,217-223$.

Schafe, G. E., Nadel, N. V., Sullivan, G. M., Harris, A., \& LeDoux, J. E. (1999). Memory consolidation for contextual and auditory fear conditioning is dependent on protein synthesis, PKA, and MAP kinase. Learning \& Memory, 6, 97-110.
Squire, L. R., \& Alvarez, P. (1995). Retrograde-amnesia and memory consolidation: A neurobiological perspective. Current Opinion in Neurobiology, 5, 169-177.

Stote, D. L., \& Fanselow, M. S. (2004). NMDA receptor modulation of incidental learning in Pavlovian context conditioning. Behavioral Neuroscience, 118, 253-257.

Sutherland, R. J., \& McDonald, R. J. (1990). Hippocampus, amygdala, and memory deficits in rats. Behavioural Brain Research, 37, 57-79.

Sutherland, R. J., Weisend, M. P., Mumby, D., Astur, R. S., Hanlon, F. M., Koerner, A., et al. (2001). Retrograde amnesia after hippocampal damage: Recent vs. remote memories in two tasks. Hippocampus, 11, 27-42.

Tan, S. E., \& Liang, K. C. (1996). Spatial learning alters hippocampal calcium calmodulin-dependent protein kinase II activity in rats. Brain Research, 711, 234-240.

Taubenfeld, S. M., Milekic, M. H., Monti, B., \& Alberini, C. M. (2001). The consolidation of new but not reactivated memory requires hippocampal C/EBP beta. Nature Neuroscience, 4, 813-818.

Teyler, T. J., \& Discenna, P. (1986). The hippocampal memory indexing theory. Behavioral Neuroscience, 100, 147-154.

Tolman, E. C. (1948). Cognitive maps in rats and men. Psychological Review, 55, 189-208.

Tronson, N. C., \& Taylor, J. R. (2007). Molecular mechanisms of memory reconsolidation. Nature Reviews Neuroscience, 8, 262-275.

von Hertzen, L. S. J., \& Giese, K. P. (2005). Memory reconsolidation engages only a subset of immediate-early genes induced during consolidation. Journal of Neuroscience, 25, 1935-1942.

Wallenstein, G. V., Eichenbaum, H., \& Hasselmo, M. E. (1998). The hippocampus as an associator of discontiguous events. Trends in Neurosciences, 21, 317-323.

Whitlock, J. R., Heynen, A. J., Shuler, M. G., \& Bear, M. F. (2006). Learning induces long-term potentiation in the hippocampus. Science, 313, 1093-1097.

Young, S. L., Bohenek, D. L., \& Fanselow, M. S. (1994). NMDA processes mediate anterograde amnesia of contextual fear conditioning induced by hippocampal damage: Immunization against amnesia by context preexposure. Behavioral Neuroscience, 108, 19-29. 